\title{
On the Method of Distant Infrared Monitoring of Forest Spaces and Gas Main Pipelines
}

\author{
R.S. Asatryan ${ }^{1}$, N.R. Khachatryan ${ }^{1}$, H.S. Karayan ${ }^{2}$ \\ ${ }^{1}$ National Institute of Metrology, Komitas ave. 49/4, 0051, Yerevan, Armenia \\ ${ }^{2}$ Yervan State University, 1 A.Manoogian str., 0025, Yerevan, Armenia
}

\begin{abstract}
The development results of a new methods of aerial (on a helicopter or airplane) infrared (IR) scanning of extensive spaces with the purpose of detecting weak heat sources (fire centers at an early stage of their development) to prevent the occurrence of large-scale fires are presented, and for probing gas main pipelines and detecting gas leaks also is described. IR scanning of pipelines was performed in the wavelength range $8-12 \mu \mathrm{m}$ by a helicopter flying along the routes of pipelines. In the paper there is presented the description of the IR radiometer as well as the measurement method of point and extended thermal sources wavelength range of 2.5 to 5.5 and 8-12 microns.
\end{abstract}

Keywords: Infrared Radiometer, Forest Fire, Natural Gas Pipelines.

\section{INTRODUCTION}

The environment monitoring, investigation and control of ecological conditions attract a great attention of the mankind, especially at the present stage of development of industry, energetic and urban building. Optoelectronic systems and devices designed for application in ecological studies and in arising extremal situations are always in the center of the scientists' and engineers' attention. In particular, research complexes for early detection of fire hearths arising during natural calamities are irreplaceable.

The main artificial source of atmospheric gaseous pollution is leakage of natural gas, in which the methane content is $95 \%$. However, the problem is aggravated by the fact that gas main pipelines (GMPs) run through sparsely inhibited and hard to reach territories, where testing is especially impeded.

It is obvious that the development of a state of the art remote and efficient method for ecological monitoring of GMPs is especially pressing. In this situation, the only practical method is remote testing from an aircraft (e.g., a helicopter) flying along the pipeline route at heights of up to $1000 \mathrm{~m}$.

The objective of this study is the description of a universal spectroradiometer (SR) we developed and demonstration that one possible field of its application is the airborne IR monitoring of GMPs.

Therefore, the development and creation of infrared devices and systems of thermal monitoring of environment, in particular, large forest spaces is a rather important problem.

The development of modern distant and effective methods of ecological monitoring of large forest spaces is more than actual. In such a situation the only method is remote monitoring from an aircraft (e.g., from a helicopter) while flying over large forests at the altitude up to $1000 \mathrm{~m}$.

\section{BRIEF TEChNiCAL DESCRIPTION OF A MEASURING SySTEM}

Structurally the measuring complex consists of two basic units: an optico-mechanical unit of the IR radiometer andan electronic control unit joined to a personal computer. It is designed to measure spectral radiance and radiation temperature (or its drops) of point and extened sources of infrared radiation under laboratory and field conditions [13]. To automate data acquisition and processing the spectroradiometer is joined to a personal computer via a seriesport RS 232. Fig.1. Optical scheme of the optico-mechanical unit (OMU) is shown in

1 Corresponding Author: as_ruben@yahoo.com 


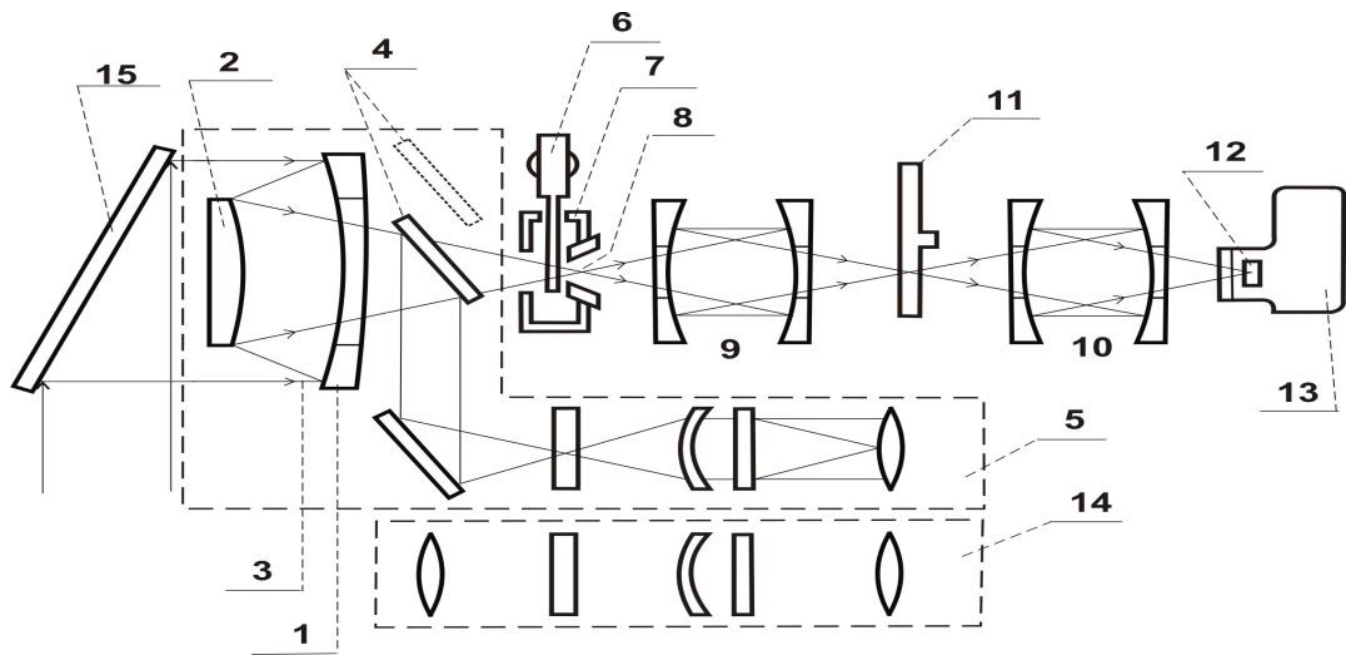

Fig1. Optical scheme of OMU.

1-Primary mirror of the objective; 2- secondary mirror of the objective; 3 - radiation from an object; 4- removable plane mirror; 5- a sight; 6- a modulator; 7- a reference cavity; 8- a field diaphragm; 9,10- projection objective; 11- a disk with interferential light filters; 12- a sensing site of the photodetector; 13- a thermos for liquid nitrogen; 14- a telescope; 15 - a deflection mirror.

$>$ Input mirror objective of Cassegrain type;

$>$ A telescope for operative pointing to an object under test, equipped with a sighting grid visible through an eyepiece on the OMU back panel;

$>$ Parallax free sight for accurate pointing the spectroradiometer to an area to be measured. The sight has a sighting grid with a cross and a circle which defines visual field boundaries of the device;

$>$ Projection objectives which serve for refocusing the radiation from a field diaphragm to the plane with light filters and to a sensing site of the photodetector. They represent pairs of spherical mirrors the application of which enables to avoid achromatic aberrations;

$>$ A block of removable ring wedge variable light filters which provide a total working spectral range of 0.4 to $14 \mu \mathrm{m}$

$>$ A photodetector which structurally represents a removable block with a photodetector placed inside it in accordance with the spectral range, a preamplifier, and an adjusting

Full working spectral range of the device is covered with the help of three sets of removable light filters and photodetectors in the subbands of 0.4 to $1.1 \mu \mathrm{m}, 2.5$ to $5.5 \mu \mathrm{m}$, and 8 to $14 \mu \mathrm{m}$. Main technical parameters of the device are given in the Table.

\begin{tabular}{|l|l|l|}
\hline \multicolumn{1}{|c|}{ № } & \multicolumn{1}{|c|}{ Parameter Name } & \multicolumn{1}{c|}{ Value } \\
\hline 1. & Input objective diameter & $180 \mathrm{~mm}$ \\
\hline 2. & Focal distance mechanism. & $200 \mathrm{~mm}$ \\
\hline 3. & Distances to be focused & from $5 \mathrm{~m}$ to $\infty$ \\
\hline 4. & Working spectral range & from 0,4 to $14 \mu \mathrm{m}$ \\
& I subband (spectral resolution of $10 \%)$ & from 0,4 to $1,1 \mu \mathrm{m}$ \\
& II subband (spectral resolution of 3\%) & from 2,5 to $5,5 \mu \mathrm{m}$ \\
& III subband (spectral resolution of $8 \%)$ & from 7,9 to $13,5 \mu \mathrm{m}$ \\
\hline 5. & $\begin{array}{l}\text { Photodetectors: } \\
\text { I subband }\end{array}$ & $\mathrm{Si}-$ photodiode \\
& $\begin{array}{l}\text { II subband } \\
\text { III subband }\end{array}$ & InSb-photoresist \\
\hline 6. & Field of vision & $3 \mathrm{mrad}$ - photoresist \\
\hline 7. & Noise equivalent difference of the radiation & $0,05 \mathrm{~K}$ \\
\hline
\end{tabular}


American Research Journal of Agriculture, Volume 1, Issue 2, April 2015

ISSN 2378-9018

\begin{tabular}{|l|l|l|}
\hline & temperatures (at $\left.295^{\circ} \mathrm{K}\right)$ & \\
\hline 8. & Continuous work time & 8 hours \\
\hline 9. & Time of preparation to work & $15 \mathrm{~min}$ \\
\hline 10. & $\begin{array}{l}\text { Dimensional size of spectroradiometer: } \\
\text { OMU } \\
\text { ECU }\end{array}$ & $\begin{array}{l}415 \times 278 \times 254 \mathrm{~mm} \\
500 \times 420 \times 210 \mathrm{~mm}\end{array}$ \\
\hline 11. & $\begin{array}{l}\text { Weight: } \\
\text { OMU } \\
\text { ECU }\end{array}$ & $\begin{array}{l}\text { not more than } 12 \mathrm{~kg} \\
\text { not more than } 15 \mathrm{~kg}\end{array}$ \\
\hline 12. & $\begin{array}{l}\text { Climatic conditions of operation: } \\
\text { Ambient temperature } \\
\text { Atmospheric pressure } \\
\text { Air relative humidity }\end{array}$ & $\begin{array}{l}\text { from }-35^{\circ} \text { to }+45^{\circ} \\
\text { from } 84 \text { to } 107 \mathrm{kPa} \text { (from } 630 \text { to } 800 \mathrm{~mm} \mathrm{Hg}) \\
\text { up to } 98 \% \text { at } 35^{\circ} \mathrm{C}\end{array}$ \\
& $\begin{array}{l}(220 \pm 22) \mathrm{V} \\
(50 \pm 1) \mathrm{Hz}\end{array}$ \\
\hline 13. & $\begin{array}{l}\text { Supply voltage frequency } \\
\text { not more than } 200 \mathrm{~W}\end{array}$ \\
\hline 14. & Power consumed & \multicolumn{2}{|c|}{} \\
\hline
\end{tabular}

During operation the OMU, by means of the wedge guide, is placed on a rotary mechanism which is fastened to the horizontal platform of a specially prepared tripod

The electronic control unit (ECU) is structurally of on-top variant. All indication and control elements are mounted on the front panel of the ECU.

Under laboratory conditions the ECU is placed on the table, and under field conditions it can be mounted in a helicopter with the help of dampers. External appearance of the units is shown in Fg.2.

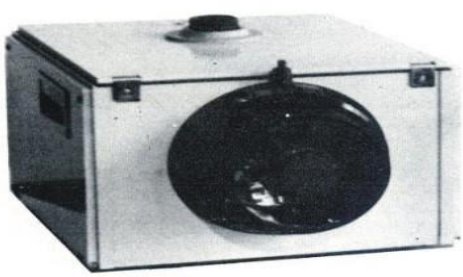

(A)

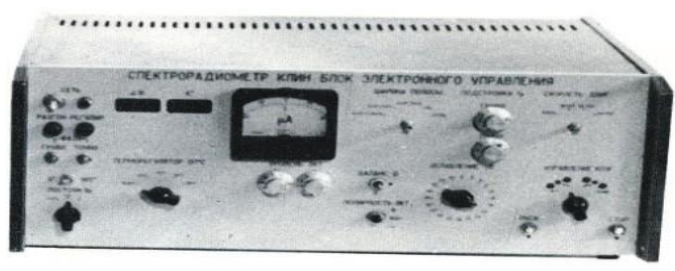

(Б)

Fig2. External appearance of the radiometer A)OMU, Б) ECU

In brief, the operation principle of the spectroradiometer consists in the following: Inside the OMU the radiation flow from the object under test is collected by means of an optical system (see Fig.1) and focused onto a sensing site of the photodetector. Further, a preamplifier amplifies an electric signal and transmits it to the ECU. In the ECU the electronic schemes amplify, demodulate and filter the signal from the photodetector output, and as a result of this there appears a signal at the output the amplitude of which is a measure of the radiation temperature of the object. Knowing the value of the collected radiation power ( through the data of peliminarily conducted energetic calibration of the device), spectral filter features of the system and amplification degree, the output signal can be exactly transformed into an absolute measurement of radiation temperatures of the objects under test.

Let's notice some advantages of the IR radiometer developed by us [4] compared to the existing close analogs. To widen functional capabilities in the sphere of spectral investigations of thermal objects, besides wideband interferential light filters for spectrum parts of 0.4 to $1.1,2.5$ to 5.5., and 8 to $14 \mu \mathrm{m}$, the device is also provided with ring readjustable light filters. To eliminate chromatic aberrations the device optical scheme includes two pairs (see Fig.1) of mirror projection objectives in the focuses of which there are placed light filters and the receiving site of photodetectors.

The IR radiometer is mounted in the helicopter and, with the help of a deflecting plane mirror, by its field of vision scans (through the bottom hatch, along the helicopter motion ruoting) terrestril surface of large forests, see Fig. 3 . 


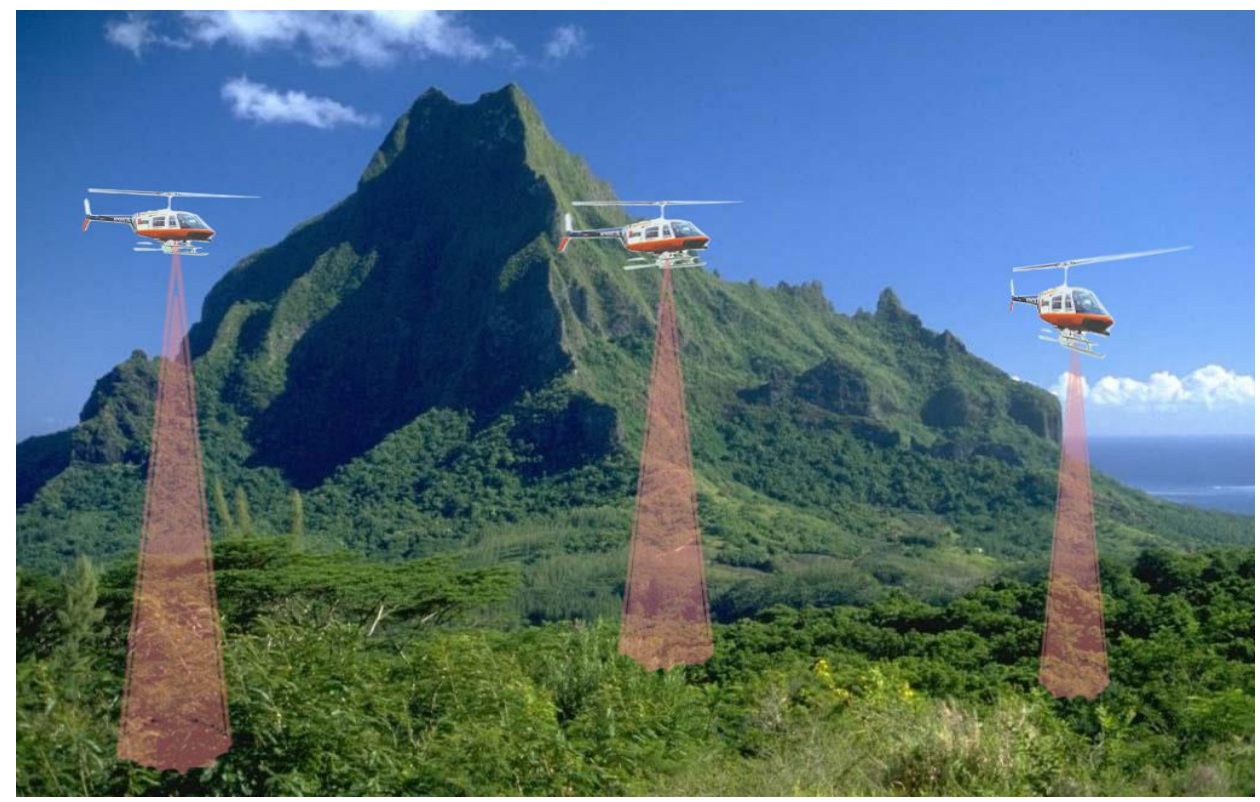

Fig3. Helicopter IR scanning of large forests.

In the presence of fire hearths the radiation temperature in this region (within the wavelength rangeof 2.5 to $5.5 \mu \mathrm{m}$ ) considerably increases that is registered by the electronic control unit.

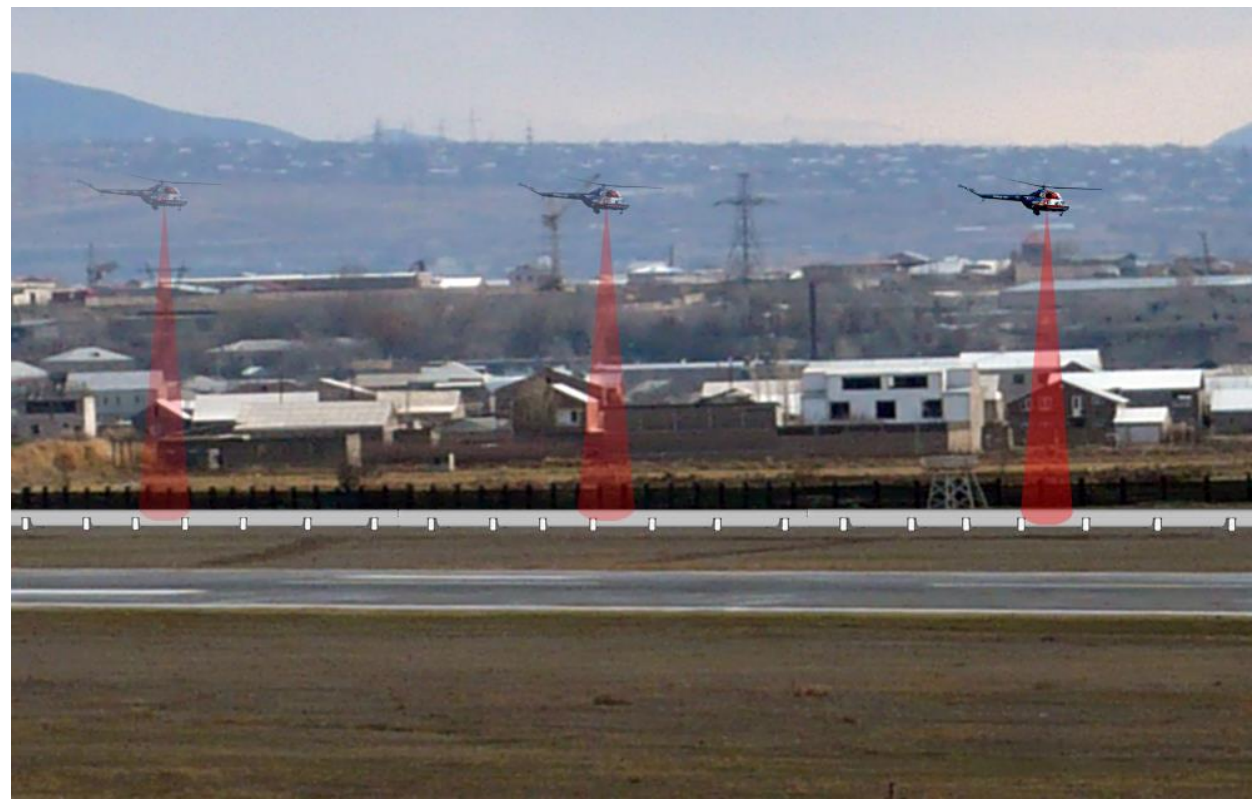

Fig4. Helicopter IR scanning of GMPs.

At the helicopter flight altitudes of 200,500 and $700 \mathrm{~m}$ the radiometer covers, with its field of vision, surface areas of about 120, 750 and 1500 sq.m, correspondingly.

The IR radiometer scans the Earth's surface along the GMPs routes within its field of view through the bottom hatch. If there are macroscopic gas leaks in this region, the radiation temperature (in the wavelength region 8-14 $\mu \mathrm{m})$ drops significantly [5] and is recorded by the ECU.

At helicopter flight altitudes of 200 and $150 \mathrm{~m}$, the radiometer fields of view on the ground encompass surfaces with radius of $\sim 6$ and $\sim 2.5 \mathrm{~m}$, respectively, see fig. 4 . 
With the helicopter speed of $150-200 \mathrm{~km} / \mathrm{hr}$ the time of one measurement cycle is $0.1 \mathrm{sec}$.

\section{Measurement TeChNiQue of IR Flows From EXTENDED AND PoINT THERMal SOURCES}

Before carrying out quantitative measurements of IR radiation emitted by an unknown source, it is necessary to fulfill energetic calibration of the spectroradiometer, the aim of which is the measurement of the device response to the known standard source (usually a black body with known temperature). By definition, the device calibration means obtaining an electrical signal at the output, which corresponds to a radiation flow unit incident into the radiometer inlet. The calibration is expressed by some function $\mathrm{k}(\lambda)$ called spectral calibration characteristic of the device, which includes combined effect of optical elements and electronic amplification of the whole system. $k(\lambda)$ is expressed in V/radiation unit, with standard level of amplification degree. An output signal of the device is proportional to the difference between the IR radiation flows coming to the photodetector from an external source and from the internal modulated reference black body. In calibrating the radiation from the calibration black body (with known temperature) entirely fills the device field of vision. An output signal $S(\lambda)$ is expressed by the following ratio:

$$
S(\lambda)=k(\lambda) \cdot\left\{r(\lambda, T) \cdot \tau(\lambda, l)-r\left(\lambda, T_{0}\right)+r\left(\lambda, T_{B}\right)[1-\tau(\lambda, l)]\right\}
$$

where $r(\lambda, T)$ is Plunk function at the temperature $T$ and the wavelength $\lambda$;

$T$ - Temperature of the calibration black body;

$\tau(\lambda, l)$ - Atmospheric transparency over the path $l$ between the calibration source and device;

$T_{0}$ - Temperature of the internal reference black body;

$T_{B}$ - Temperature of the air during the experiment.

In the windows of the atmosphere transparency (e.g. for the wavelength range of 2.5 to $5.5^{\mu} \mathrm{m}$ ), where the transmission is high, $\tau(\lambda, l)$ may be taken as 1 , if the calibration is carried out from the distance " $l$ " equal to several meters. Therefore in this approximation for $S(\lambda)$ we can write:

$$
S(\lambda)=k(\lambda) \cdot\left[r(\lambda, T)-r\left(\lambda, T_{0}\right)\right]
$$

With the amplification coefficient equal to 1 . And in measuring with the amplification coefficient different from 1 the $S(\lambda)$ value decreases by the same factor. The Plunk function value is calculated according to the ratio:

$$
r(\lambda, T)=\frac{c_{1}}{\lambda^{5}}\left[\exp \left(c_{2} / \lambda T\right)-1\right]^{-1}
$$

Where

$$
\begin{aligned}
& c_{1}=3,74-10^{4} \mathrm{~W} \mu \mathrm{m}^{4} / \mathrm{cm}^{2} \\
& c_{2}=1,438 \cdot 10^{4} \mu \mathrm{m} \mathrm{deg}
\end{aligned}
$$

The objects studied the radiation flow of which completely fills the device field of vision are extent in these measurements. In this case radiance spectral density (W $(\lambda, T)_{\mathrm{W} / \mathrm{cm} 2} \mu_{\mathrm{m}) \text { of the object is measured. The ratio }}$ (1) may be rewrite as:

$$
S(\lambda)=k(\lambda)\left\{W(\lambda, T) \cdot \tau(\lambda, l)-r\left(\lambda, T_{0}\right)+r\left(\lambda, T_{B}\right)[1-\tau(\lambda, l)]\right\} \cdot \beta
$$

Where $W(\lambda, T)$ the radiance spectral density of the object studied is, $\beta$ is an amplification coefficient of the whole system, and the rest symbols remain previous. The atmosphere transparency $\tau(\lambda, l)$ is either measured simultaneously, or calculated with the help of data from literature [5,6]. From the ratio (3) we can get for $W(\lambda, T)$ 


$$
W(\lambda, T)=\frac{S(\lambda) / k(\lambda) \beta+r\left(\lambda, T_{0}\right)-r\left(\lambda, T_{B}\right) \cdot[1-\tau(\lambda, l)]}{r(\lambda, l)}
$$

Usually the radiation of point sources does not fill the visual field of the device. If the area A of a radiating object is known we can measure its spectral radiance according to the above-stated technique, that is

$$
W_{p}(\lambda, T)=W(\lambda, T) \cdot \omega \cdot \frac{l^{2}}{A}
$$

Where $\omega$ is a solid angle of the spectroradiometer visual field, $\mathrm{W}(\lambda, \mathrm{T})$ is a total spectral radiance measured according to (4); $l$ is the distance from the object under test to the spectroradiometer. While measuring point sources spectral contrast of a radiation source is also of interest, when the background radiance is comparable to the object radiation. In this case it is necessary to separate the background signal $S_{\Phi}(\lambda)$ from the signal "source+background" $S(\lambda)$. For the spectral radiation contrast of the source we can get the ratio:

$$
W(\lambda)=\frac{\Delta S(\lambda) \cdot \omega \cdot l^{2}}{\beta \cdot k(\lambda) \cdot \tau(\lambda, l) A}
$$

Where $\Delta S(\lambda)=S(\lambda)-S_{\Phi}(\lambda)$

If $\mathrm{A}$ is unknown we may define the contrast of the spectral luminous intensity of the source (in $\mathrm{W} / \mathrm{strad} . \mu \mathrm{m}$ ):

$$
I(\lambda)=W(\lambda) \cdot A=\frac{\Delta S(\lambda)}{\beta \cdot k(\lambda) \cdot \tau(\lambda, l)} \cdot \omega \cdot l^{2}
$$

Calculation of the radiation temperatures of the objects under test is carried out in accordance with specially developed algorithms and programs.

\section{CONCLUSION}

Application of the given method of remote ecological monitoring of vast forest spaces and extended gas pipelines will undoubtedly bring to the considerable technical-economical effectiveness and will also have a great importance in the problem of preventing the fire occurrences, especially of large-scale ones, and also will be imported in solving the problem of monitoring atmospheric pollution from natural - gas emissions.

\section{REFERENCES}

[1] R.S Asatryan, Optoelectronic Methods and Means of Intesting Physico-Ecological Parameters of Atmosphere and Thermal Objects, News of NAS RA, Techn. Sciences, Vol. LX, No. 2, (2007) 307(in Russian).

[2] R.S Asatryan, S.R Asatryan, et al., Infrared Scanning Complex for Preventing Large-Scale Fires, Ecological Systems and Devices, No. 1,(2008) 17(in Russian).

[3] R.S. Asatryan et al. Universal Infrared Spectral Radiometer. Intern. Journal of IR and MM Waves, vol . 24, No. 6..(2003), 1035 .

[4] R.S. Asatryan, H. G. Gevorgyan et al. The Spectroradiometer, Patent of Ra, No.1678A2, Appl. No. P20050042, 11.04.2005.(in Armenian).

[5] Shilin, B.V. and Molodchinin, I.A., Kontrol' sostoyaniya okruzhayushchei sredy teplovoi aeros"emkoi (Monitoring of Environment by IR Aerial Survey), Moscow: Nedra, 1992.

[6] P. Kruz, L.Macgloulin ,P. Maccvistan , Bases of Infrared Technique, M. Voenizdat, USSR DD, 1964, 464p.(in Russian).

[7] A.V. Pavlov, Optoelectronic Devices, M. Energia, 1974, 360p(in Russian). 